\title{
On the Wake Flow Interaction between Model Turbines with Varying Streamwise Distance
}

\author{
Mubashar Khan1, Ylva Odemark2 ${ }^{2}$ Mats Sandberg1, Jens H. M. Fransson ${ }^{1,3}$ \\ ${ }^{1}$ Energy Systems, University of Gävle, Gävle, Sweden \\ ${ }^{2}$ Vattenfall AB, Stockholm, Sweden \\ ${ }^{3}$ Dept. Mechanics, Royal Institute of Technology, Stockholm, Sweden \\ Email:mubashar.khan@hig.se,jens.fransson@hig.se,ylva.odemark@vattenfall.com,jensf@kth.se
}

How to cite this paper: Khan, M., Odemark, Y., Sandberg, M. and Fransson, J.H.M. (2017) On the Wake Flow Interaction between Model Turbines with Varying Streamwise Distance. Open Journal of Fluid Dynamics, 7, 557-578.

https://doi.org/10.4236/ojfd.2017.74038

Received: October 17, 2017

Accepted: December 16, 2017

Published: December 19, 2017

Copyright $\odot 2017$ by authors and Scientific Research Publishing Inc. This work is licensed under the Creative Commons Attribution International License (CC BY 4.0).

http://creativecommons.org/licenses/by/4.0/ Open Access

\begin{abstract}
Wind tunnel measurements using particle image velocimetry have been performed around two perforated discs, with varying streamwise distance, in order to simulate the wake interaction between wind turbines. The static pressure footprint ( $\mathrm{p}-\mathrm{f}$ ) on ground level associated with the wake behind the disc and wake velocity data for both the streamwise and wall-normal velocity components with the corresponding turbulence intensities are reported. The p-f method shows that the size of the wake regions, behind the wind turbine models, initially drop when a second disc is placed just downstream of the first one. From a mutual distance ( $\Delta x)$ of about five disc diameters $(5 D)$, both wake footprints increase as the mutual distance is increased, and for very large mutual distances, approximately $\Delta x / D>15$, the footprint of the downstream disc has recovered and is about the same as for a single disc. At last we conclude that despite very different inlet conditions to the discs, with about $50 \%$ of reduced velocity on the centre line upstream of the second disc and an increase of the maximum streamwise fluctuations by $90 \%$, the mean velocities in the wake are proven to scale with the hub height velocity.
\end{abstract}

\section{Keywords}

Wind Turbine Model, Perforated Disc, Wake Flow Interaction, Particle Image Velocimetry, Pressure Footprint

\section{Introduction}

Inside a wind power farm, the flow field is strongly modified compared to the undisturbed flow. Behind each turbine there is a velocity deficit, which reduces the power output for the downwind turbines [1] [2]. Furthermore, the increased 
turbulence levels in the wakes cause an enhanced risk for structural fatigue [3]. Knowledge about the flow field inside wind power farms is therefore important for power production estimations and life time predictions of the individual turbines. For an industrial application, fully resolved numerical simulations of the flow field around a whole wind farm is still not feasible due to the computational cost, especially if different wind speeds and directions are of interest. The flow and the turbines therefore need to be modelled in some way, in order to reduce the computational time of the simulations. A widely used model is the actuator disc (ACD) model, where the rotor is replaced by a drag force, distributed across the rotor disc area. This method saves an extensive amount of computational time, as compared to modeling the whole turbine and the rotating blades. The ACD model and its experimental counterpart, namely the porous disc model, have been used extensively to simulate the flow around wind turbines (see e.g. [4] [5] [6] [7]). Cal et al. [8] performed a study with 9 rotating turbine models placed in a boundary layer of a wind tunnel in order to study the mean velocity and turbulence properties, as well as the corresponding impact on the vertical transport of kinetic energy. España et al. [9] [10] carried out experiments with a single porous disc, placed in both an atmospheric boundary layer flow and in conditions of homogeneous isotropic turbulence, with focus on the random displacements of the wake centre. The horizontal displacements of the wake were found to be larger than the vertical ones in the case with atmospheric boundary layer flow. A direct experimental comparison of the wake properties between a rotating three-bladed wind turbine model and a porous disc was reported by Aubrun et al. [11]. In the modeled atmospheric boundary layer the two wakes are indistinguishable three rotor diameters downstream of the models. This result strongly supports wind turbine interaction studies using porous disc models.

The flow around a porous disc differs from that around a solid disc. For the perforated disc, the interaction between the separated shear layers from the edges of the disc is disturbed by the flow through the disc, which gives the perforated disc an increased pressure behind the disc and thus a lower drag as compared to the solid disc. The formation region of the vortices is also larger, which gives the porous disc a higher Strouhal number as compared to the solid disc, as shown by Castro [12] for perforated plates of varying solidity at Reynolds numbers $R e \sim 10^{4}$. The drag force naturally increases with an increasing solidity. For a porous disc, the vortex shedding depends on the solidity and a disc with a solidity lower than a certain threshold does not experience any shedding. Cannon et al. [13] performed flow visualizations behind porous discs with $50 \%, 60 \%$ and $85 \%$ solidity and for a solid disc. The shedding was very clear for the solid disc and the $85 \%$ case. Miau et al. [14] measured the shedding frequency behind discs at Reynolds numbers between $10^{3}$ and $10^{5}$. The Strouhal number was found to be around 0.14 and slightly increasing with the Reynolds number. It was concluded that the anti-phase characteristics of positions located $180^{\circ}$ apart were preserved, but that the shedding also had a random nature. 
Experiments on a disc with approximately the same solidity and thrust coefficient as the ones used in the present experiment have previously been carried out by Medici [15]. The Strouhal number was there found to be in the range $0.12-0.14$ and a similar Strouhal number is expected for the present turbine models. This feature was however not studied in the present work. The wind turbine model by means of a porous disc implies some limitations on describing the physical process on the wake generation, both the presence of tip vortices and the rotation of the wake behind real wind turbines is completely bypassed. But a meticulous selection of the solidity of $58 \%$ provide the same coefficient of thrust as the rotating counterparts and the thrust effect is the major factor influencing the wake characteristics [16].

Although a number of numerical and experimental studies have been performed with turbines modelled as porous discs, there is still a need for additional experimental data, both for gaining an increased understanding of wake interaction and for validation of numerical studies.

In the present paper we present static pressure and velocity data obtained with a pressure plate [17] and particle image velocimetry (PIV), respectively. Two identical porous discs were used, which were placed with the mutual streamwise distances of 4 and 5 disc diameters. As a reference, measurements were also carried out on a single disc. Data of mean velocity in the streamwise and vertical directions are presented, as well as the corresponding turbulence intensities.

\section{Experimental Setup}

\subsection{Wind Tunnel and Measurement Techniques}

The measurements were performed in the atmospheric wind tunnel at the University of Gävle. The wind tunnel facility is a closed loop tunnel with a total length of $28 \mathrm{~m}$ and a maximum velocity of $22 \mathrm{~m} / \mathrm{s}$. The contraction ratio is 3:1 and the tunnel is operated by a $45 \mathrm{~kW}$ motor driving two parallel fans, which are placed in the return circuit. The tunnel is equipped with guiding vanes and honeycombs to improve the flow quality. The size of the test section is $11 \times 3 \times 1.5 \mathrm{~m}^{3}$ (length $\times$ width $\times$ height).

The hub height $(H)$ and diameter $(D)$ of the discs were both $45 \mathrm{~mm}$. The solidity of the discs was $56 \%$ and the thrust coefficient was 0.85 , which was measured with a force balance. The present thrust coefficient $\left(C_{T}\right)$ is in the upper range of real wind turbines and is defined as

$$
C_{T}=\frac{T}{0.5 \rho U_{\text {hub }}^{2} A},
$$

where $T$ is the total thrust force, $\rho$ is the air density, $U_{\text {hub }}$ is the velocity at hub height and $A=\pi D^{2} / 4$ is the disc area. A picture of one of the discs is shown in Figure 1. A right-handed coordinate system is used, with $x$ as the streamwise coordinate (positive downstream), $z$ as the vertical coordinate (positive upwards) and $y$ as the spanwise coordinate. The origin is placed at the centre of the most upstream disc. The mean streamwise velocity is denoted by $U$ 


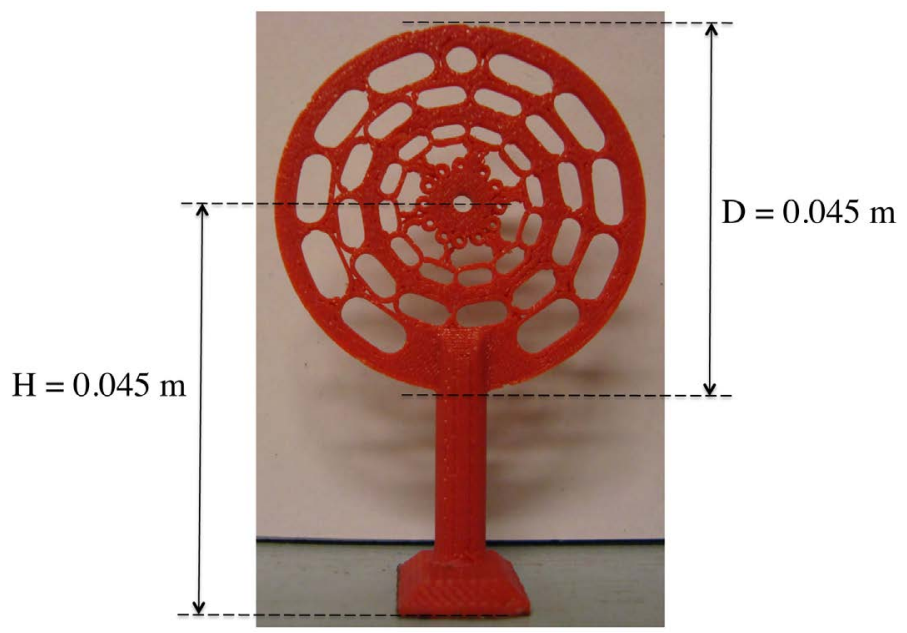

Figure 1. Picture of one of the discs used in the present experiment.

and the root-mean-square value of the fluctuations with $u_{\mathrm{rms}}$. The corresponding notation for the vertical component is $W$ and $w_{\mathrm{rms}}$.

The pressure plate is a flat square metallic surface with $400(20 \times 20)$ uniformly distributed pressure taps. The taps are connected to a pressure transducer via an in-house built multi-pressure scanner system, and the data acquisition was done using LabView via a National Instrument data acquisition card. The pressure at the hub height was measured using a Prandtl tube. The static pressure at the surface was measured and statistically averaged over a period of 25 seconds for each tap. All the results presented from the static-pressure measurements in the present paper were taken at a free-stream velocity of $U_{\infty}=16 \mathrm{~m} / \mathrm{s}$.

Pressure footprints are plotted as contour plots, in non-dimensional form, as the pressure coefficient $C_{p}$ defined as

$$
C_{p}=\frac{\Delta p}{q_{\infty}}-\mathcal{B}
$$

The dynamic pressure $\left(q_{\infty}\right)$ is in turn defined as

$$
q_{\infty}=p_{0}-p_{\infty}=\frac{1}{2} \rho U_{\infty}^{2},
$$

where $p_{0}$ and $p_{\infty}$ are the total and static pressures from a Prandtl tube mounted upstream of the turbine model in the ceiling and $\rho$ is the air density. The pressure difference $\Delta p$ in Equation (2) corresponds to

$$
\Delta p=p_{i}-p_{\infty},
$$

where $p_{i}$ is the static pressure at the $i^{\text {th }}$ pressure tap. $\mathcal{B}$ in Equation (2) corresponds to

$$
\mathcal{B}=p_{i}^{\text {clean }} /\left(1 / 2 \rho_{\infty} U_{\infty}^{2}\right),
$$

where $p_{i}^{\text {clean }}$ is the clean plate static pressure. This means that the $C_{p}$ shift in terms of $\mathcal{B}$, in Equation (2), is a weak function of $U_{\infty}$. As long as $U_{\infty}$ is 
constant $\mathcal{B}$ will not change.

The actual size of the pressure footprint, i.e. the area enclosed by a specific contour line using the $C_{p}$ distribution, is normalized by $D^{2}$.

For the PIV measurements, a HI Sense Mk II camera was used, with a total number of $1344 \times 1024$ pixels. The physical size of each image was $0.103 \times 0.078$ $\mathrm{m}^{2}$ for the reference case with one disc and $0.097 \times 0.074 \mathrm{~m}^{2}$ for the measurements with two discs. A total number of 1000 image pairs were acquired at each measurement position. For the evaluation, an adaptive correlation scheme was used, with $50 \%$ overlap and an interrogation area of $32 \times 32$ pixels. The sampling frequency was $6.1 \mathrm{~Hz}$. The mean velocities and standard deviations are scaled with the velocity at hub height in absence of the discs, which was $U_{\text {hub }}=7.0 \mathrm{~m} / \mathrm{s}$. The corresponding free-stream velocity for the PIV measurements was $U_{\infty}=8.8 \mathrm{~m} / \mathrm{s}$ and the boundary layer thickness $\left(\delta_{99}\right)$ was about $0.2 \mathrm{~m}$ at the location of the measurements. This gives $\delta_{99} / H \approx 4.4$, which models a suburb terrain with a typical gradient height of $360 \mathrm{~m}$ [18].

The reason for the free-stream velocity mismatch between the pressure measurements and the velocity measurements is related to the PIV image quality, which improved significantly at lower velocities. This was however realized after the pressure measurements, which was performed before the PIV measurements, and hence not repeated. However, for the present investigation where we only seek qualitative results using this pressure footprint method we believe that the mismatch of the free-stream velocity giving rise to a relatively small change in Reynolds number is of negligible importance.

\subsection{Flow Configurations}

Three different disc configurations (C1-C3) were investigated, which are summarized in Table 1. Recalling from Section 2.1 that the origin is placed at the centre of the most upstream disc, leaves the locations of the disc centers at $(x / D, y / D, z / D)=(0,0,0)$ and $(x / D, y / D, z / D)=(5,0,0)$ for the C2 case, and at $(x / D, y / D, z / D)=(0,0,0)$ and $(x / D, y / D, z / D)=(4,0,0)$ for the C3 case.

For the $\mathrm{C} 1$ case, measurements were performed in the region $-0.4 \leq x / D \leq 5.9$ at 14 different spanwise positions located between $0 \leq y / D \leq 3.6$. The total covered distance of $6.3 \mathrm{D}$ in the streamwise direction corresponded to three different positions for the camera and laser, with a few millimetres of overlap between the measured planes. The vertical extent of each plane was

Table 1. Summary of the three disc configurations. C1: 1 disc (reference case), C2: 2 discs 5D apart, C3: 2 discs $4 D$ apart.

\begin{tabular}{|c|c|c|c|}
\hline Case & $x$ domain & $y$ positions $(y / D)$ & $z$ domain \\
\hline $\mathrm{C} 1$ & $-0.4<x / D<5.9$ & {$\left[\begin{array}{llllll}0 & 0.2 & 2.0 & 2.5 & 3.0 & 3.6\end{array}\right]$} & $-0.7<z / D<1.0$ \\
\hline $\mathrm{C} 2$ & $-0.3<x / D<7.4$ & {$\left[\begin{array}{lllllll}0 & 0.3 & 0.5 & 0.6 & 0.7 & 1.1\end{array}\right]$} & $-0.6<z / D<1.1$ \\
\hline $\mathrm{C} 3$ & $1.7<x / D<7.9$ & {$\left[\begin{array}{lllllll}0 & 0.3 & 0.5 & 0.6 & 0.7 & 1.1\end{array}\right]$} & $-0.6<z / D<1.1$ \\
\hline
\end{tabular}


$-0.7 \leq z / D \leq 1.0$.

For the C2 and C3 cases measurements were performed in the streamwise ranges $-0.3 \leq x / D \leq 7.4$ and $1.7 \leq x / D \leq 7.9$, respectively. Four and three different positions for the camera and laser were applied for C2 and C3, respectively, with a few millimetres of overlap between the measured planes, in order to cover the whole streamwise extents. The vertical extent was $-0.6 \leq z / D \leq 1.1$ in both cases. Sketches of the setup for all three cases are shown in Figure 2, with the measurement regions and spanwise positions being summarized in Table 1.

\section{Pressure Measurements}

An example of the pressure footprint behind a single turbine model located at $(x / D, y / D)=(0,0)$ is shown in Figure 3(a). The white solid line corresponds to a pre-selected contour level value $\xi_{c_{p}}$. This value corresponds to $35 \%$ of the

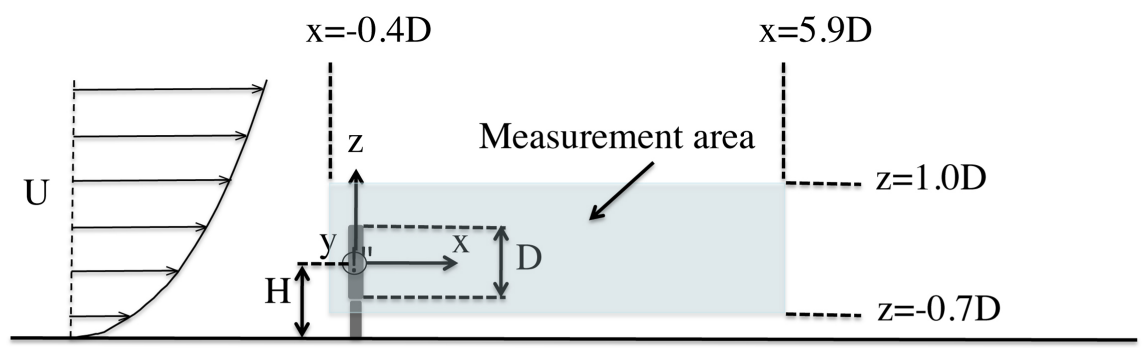

(a)

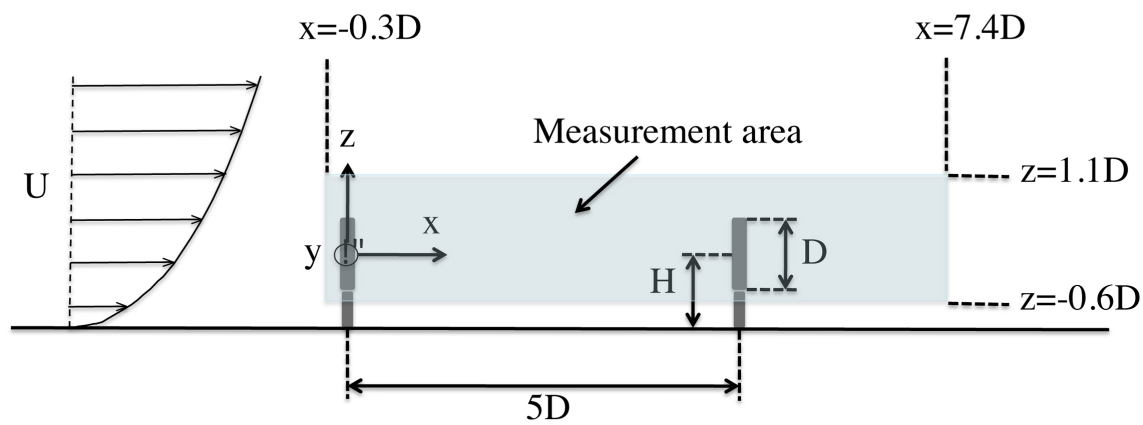

(b)

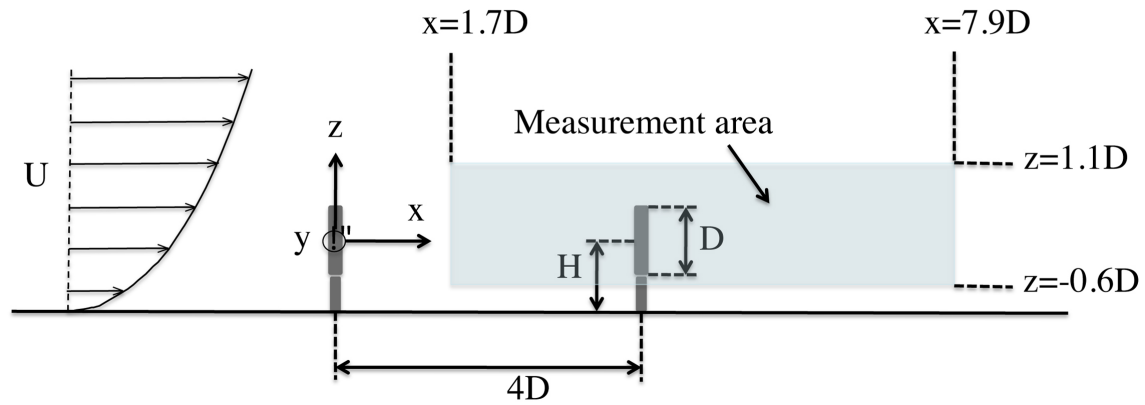

(c)

Figure 2. Sketch of the setup and the measurement regions for (a) C1, (b) C2 and (c) C3. $D=0.045 \mathrm{~m}$ and $H=0.045 \mathrm{~m}$. 

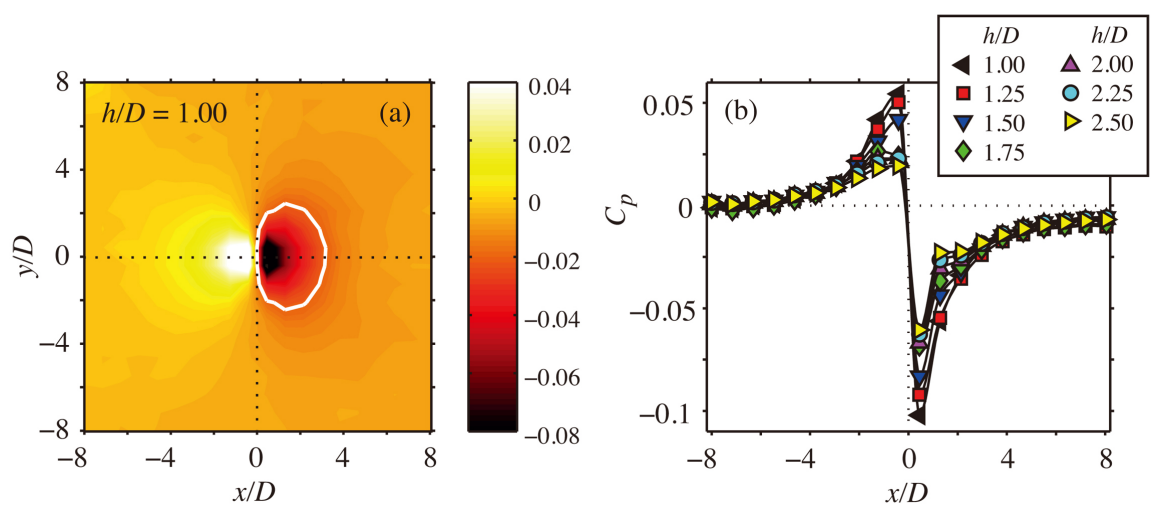

Figure 3. (a) Pressure footprint as a contour plot of $C_{p}$ for s single turbine model at hub height $h / D=1.0$. (b) Centreline pressure distribution for varying $h / D$. The white contour line in (a) corresponds to $\xi_{c_{p}}=-0.02$. Flow is from left to right in the figures.

$\max \left\{C_{p}\right\}$-value of the $C 1$ case with hub height $h / D=1.0$, where $\max \left\{C_{p}\right\}=0.058$, i.e. a contour level value of $\xi_{c_{p}}=-0.02$. Here, the minus sign, simply indicates that it is the pressure footprint in the wake of the porous disc, which is of interest.

In Figure 3(a) the flow is from left to right and shows a high pressure zone upstream of the porous disc due to the blockage of flow resulting in a retardation of the velocity and hence an increase in pressure. Behind the disc the presence of the wake is also clearly shown as a low pressure footprint, which is emphasized by the white contour line as an indicator and measure of the wake size when compared with different flow configurations. One may observe that the pressure recovery is relatively fast, already at $x / D=4$ the pressure has almost recovered rendering a uniform and close to zero-level contour map downstream of this location. The method, which we refer to as the pressure footprint ( $\mathrm{p}-\mathrm{f}$ ) method, gives a detailed picture of the near wake. In Figure 3(b) the $C_{p}$ distribution on the centre line across the porous disc is shown for different hub heights $(h / D)$. Both the maximum and the minimum $C_{p}$ values decrease as the hub height is increased, resulting in a weaker pressure footprint. In analogy, a lower or a higher free-stream velocity would be manifested in the same way, i.e. with a weaker and a stronger pressure footprint, respectively, since the disc is located inside the boundary layer.

For the analysis of the interaction between two wind turbines using the p-f method, two porous discs were positioned on the same centre line with variable streamwise distance $(\Delta x / D)$. In Figures $4(\mathrm{a})$-(d) contour plots of $C_{p}$ are shown with increasing relative distance $(\Delta x / D)$ between the upstream and downstream wind turbine model. The white contour lines correspond to the level $\xi_{c_{p}}=-0.02$. When a second disc is added closely behind the first disc one may observe that the wake footprints, both of the upstream and the downstream wind turbine, initially drop in size with respect to a single wind turbine. For a mutual distance in the range $\Delta x / D \lesssim 5$ the wake size ratio of the two models is 

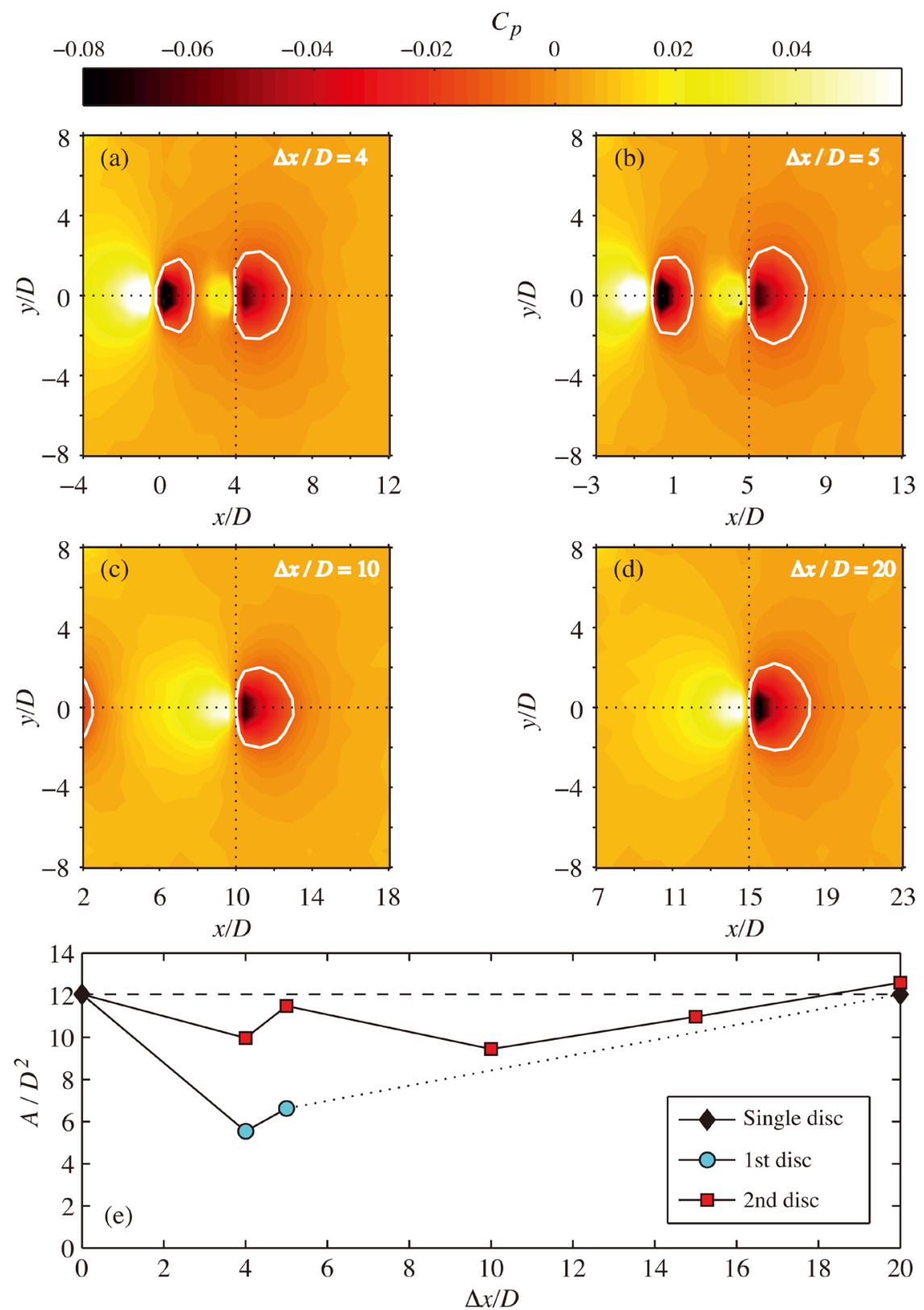

Figure 4. (a)-(d) Show the effect on the pressure footprint with successively increasing the mutual streamwise distance between two discs $\Delta x / D=4,5,10,15$. The white contour lines correspond to the level of $\xi_{c_{p}}=-0.02$. Flow is from left to right in the figures. (e) Shows the size of the wake footprint, corresponding to the area of the white contour lines, for the first and the second disc. The horizontal dashed line corresponds to the footprint area of a single disc. The dotted line indicates the expected trend of the wake size behind the upstream disc.

about two, with the downstream one being larger than the upstream one. Thereafter the wake footprints increase in size as the mutual distance is increased, and for very large mutual distances, approximately $\Delta x / D>15$, the footprint of the downstream wind turbine has recovered and is about the same as a single wind turbine. This result is quantified in Figure 4(e). 


\section{Velocity Measurements}

\subsection{Inflow Conditions}

The inflow conditions were measured with hot-film anemometry without the presence of discs. The measurements were taken in the middle of the test section, at $x / D=0$. The hot-film data was used to validate the PIV data, which showed good agreement. The results for the mean streamwise velocity and the streamwise turbulence intensity are shown in Figure 5(a) and Figure 5(b), respectively, with close ups of the near-wall region in Figure 6. Note that the discs are spanning the vertical range $-0.5 \leq z / D \leq 0.5$.

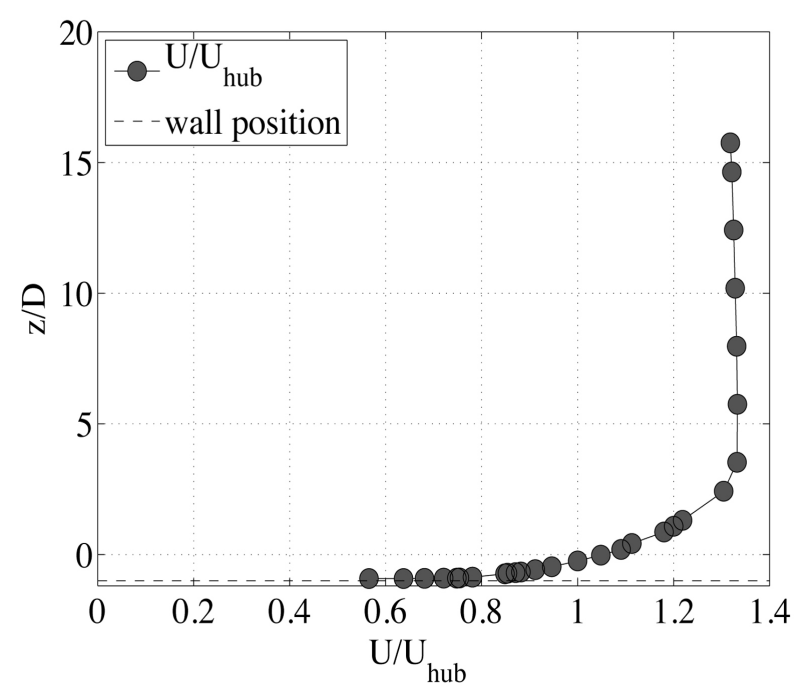

(a)

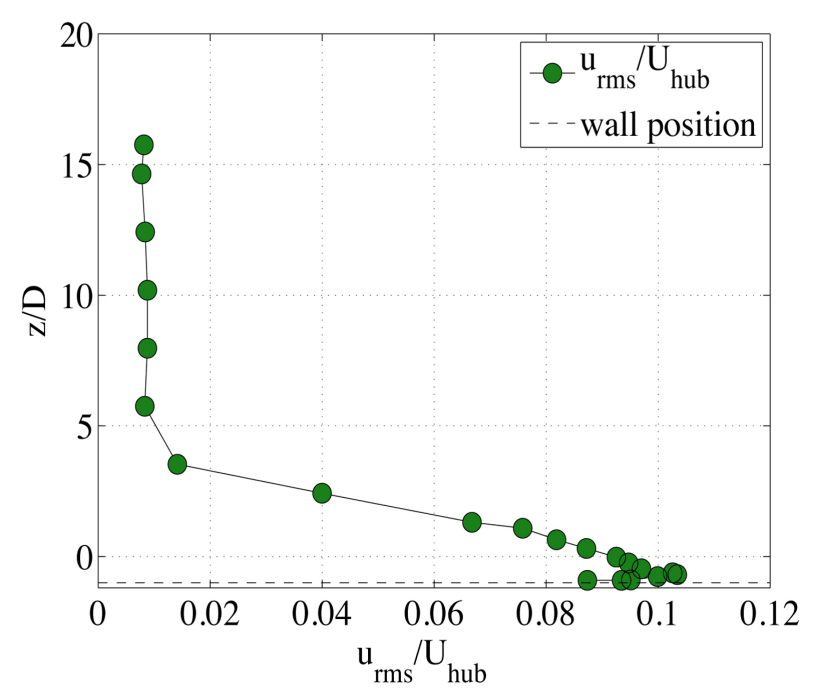

(b)

Figure 5. The inlet conditions for the present setup, measured at $x / D=0$. (a) Mean streamwise velocity, (b) streamwise turbulence intensity.

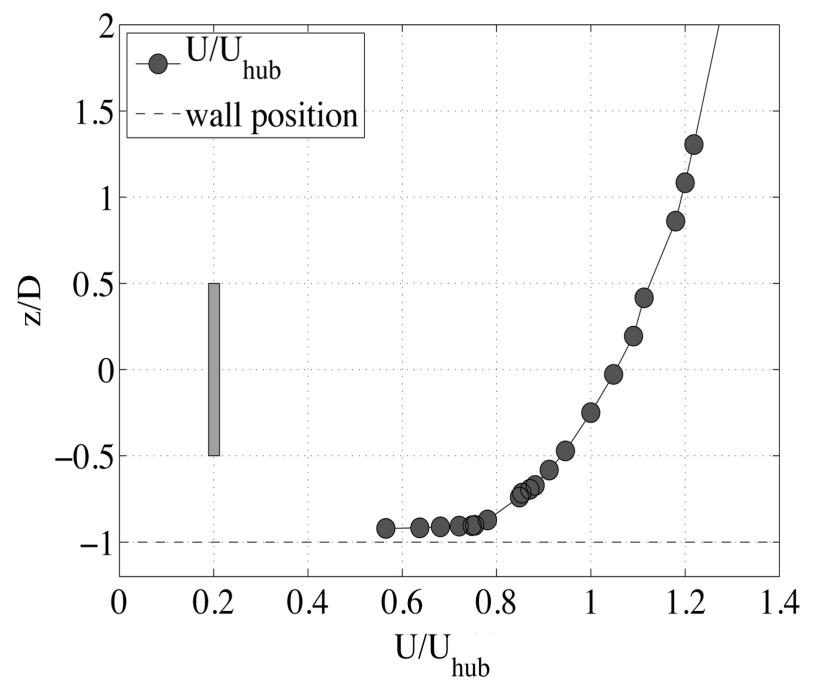

(a)

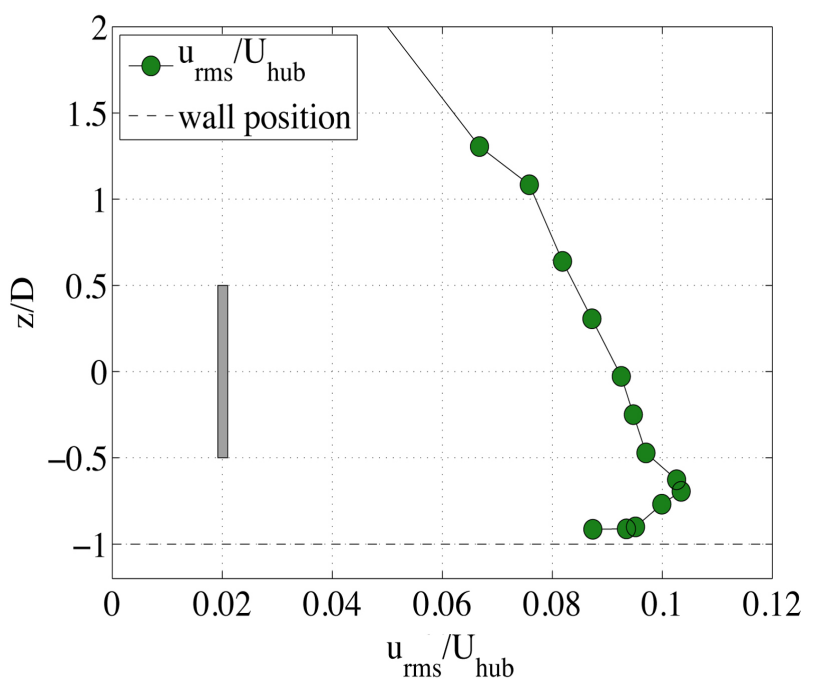

(b)

Figure 6. Same data as in Figure 5, but with a different scale on the ordinate. The disc location in the boundary layer is illustrated with a grey rectangle. 


\subsection{C1: Reference Case with 1 Disc}

Cross-sectional planes of the mean streamwise velocity for the $\mathrm{C} 1$ case are shown in Figure 7 with dashed lines indicating the location of the porous disc. For $x=1 D$ (Figure $7(\mathrm{a})$ ), it can be seen that the disc location corresponds approximately to the contour line $U / U_{\text {hub }}=0.6$. At $x=3 D$, the disc location corresponds to the contour line $U / U_{\text {hub }}=0.7$, and for $x=5 D$ to 0.75 . The wake spreading can be compared to one-dimensional momentum theory, which states the following relationships between velocity at the disc $U_{d}$, hub velocity $U_{\text {hub }}$, velocity in the wake $U_{w}$, thrust force $T$ and mass flow rate $\dot{m}=\rho U_{d} A$ :

$$
U_{d}=\frac{U_{\text {hub }}+U_{w}}{2}, \quad T=\dot{m}\left(U_{\text {hub }}-U_{w}\right)
$$

By combining these two equations and using $U_{\text {hub }}=7.0 \mathrm{~m} / \mathrm{s}$ and thrust coefficient $C_{T}=0.85$, the scaled disc velocity is calculated to $U_{d} / U_{\text {hub }}=0.7$ and the scaled wake velocity to $U_{w} / U_{\text {hub }}=0.4$. This suggests a faster spreading of the wake as compared to the experimental data shown in Figure 7. According

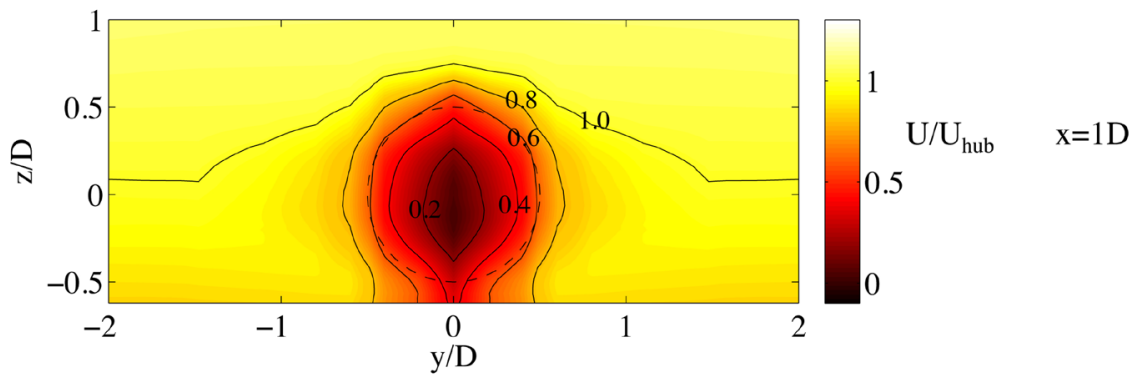

(a)

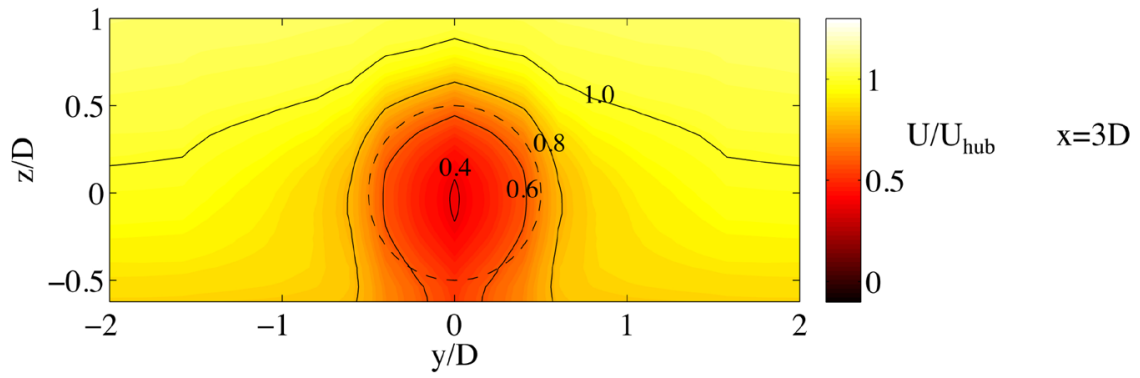

(b)

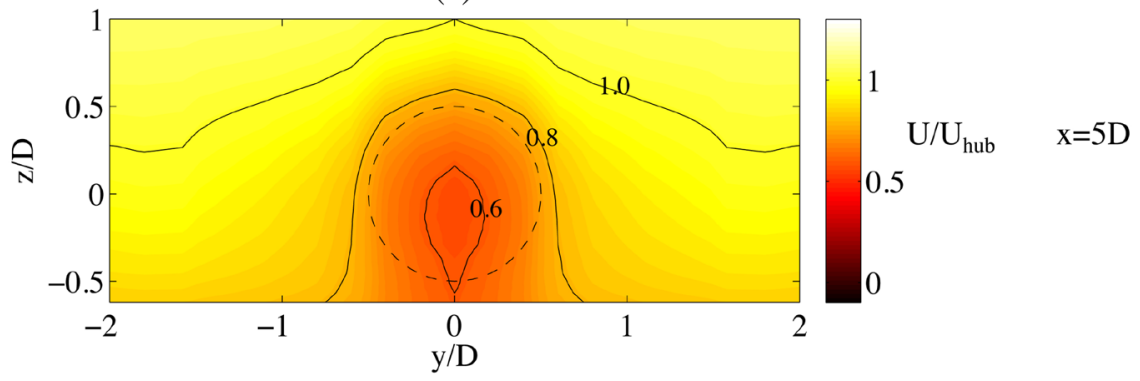

(c)

Figure 7. Case C1. Mean streamwise velocity behind the disc. The positions are from top to bottom: (a) $x=1 D$, (b) $x=3 D$ and (c) $x=5 D$. The dashed circles show the disc location. 
to this theory, a wake velocity of $U_{w} / U_{\text {hub }}=0.4$ would in this case correspond to a wake radius of $R_{w} / D=0.7$.

The wake is also shown for different spanwise positions in Figure 8. Five different spanwise positions are shown, ranging from $y=0$ to $y=0.8 D$. A few contour lines have been marked as black lines in the figure, and the location of the disc has been marked with a grey rectangle. On the centre line $(y=0)$, a large velocity deficit can be seen, where the minimum velocity at $1 D$ behind the disc is approximately $0.1 U_{\text {hub }}$ (Figure $8(a)$ ). The minimum velocity on the wake center line is then gradually increased and at $y=0.8 D$, only a weak sign of the wake is left in the form of a negative velocity gradient in the streamwise
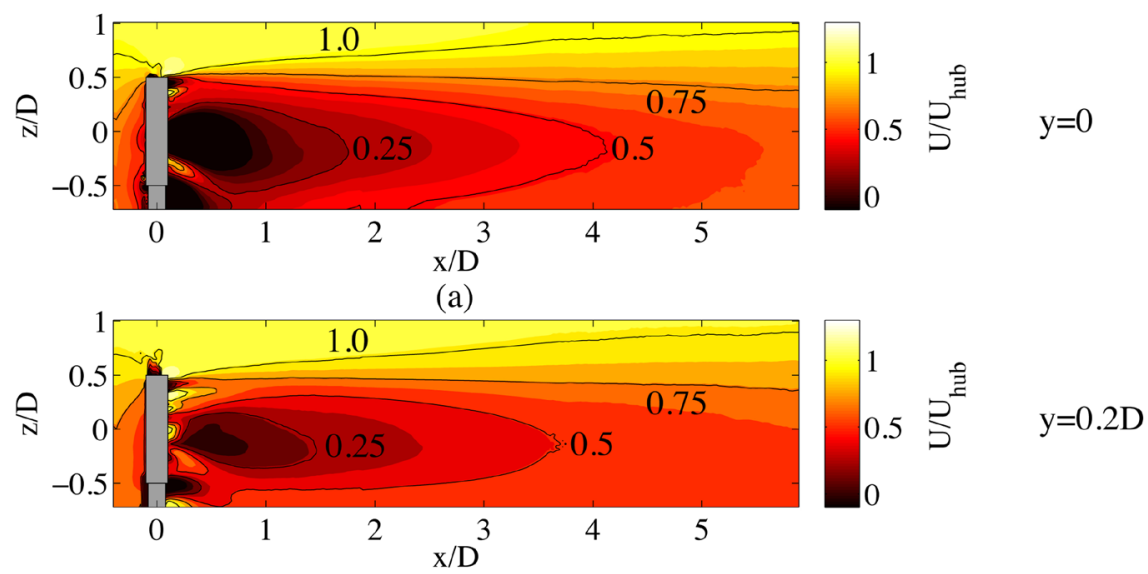

(b)

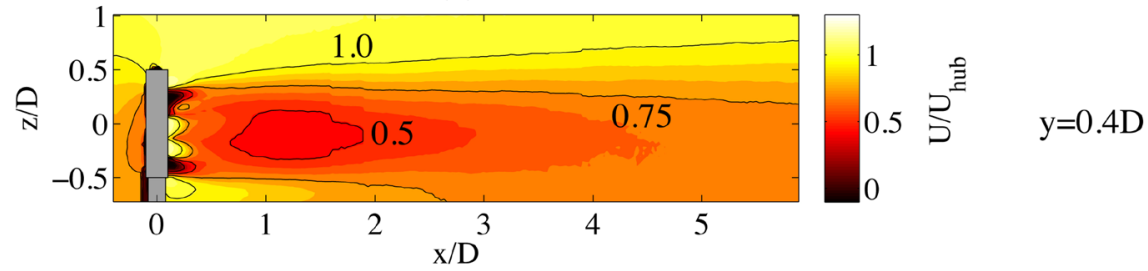

(c)

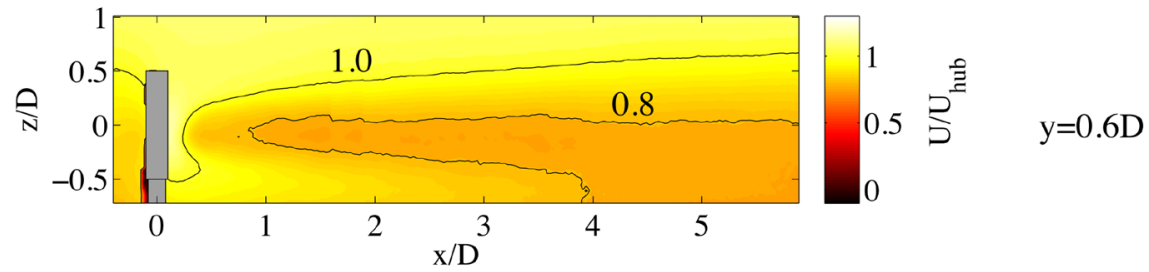

(d)

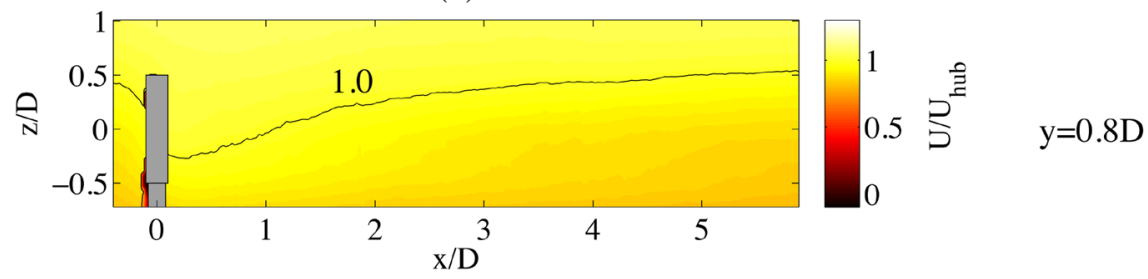

(e)

Figure 8. Case $\mathrm{C} 1$. Mean streamwise velocity behind the disc. The spanwise positions are (a) $y=0$ (straight behind the disc), (b) $y=0.2 D$, (c) $y=0.4 D$, (d) $y=0.6 D$ and (e) $y=0.8 D$. The grey rectangle show the disc location. 
direction, i.e. a sign of the spreading of the wake. At $y=1 D$ (not shown here) the trace from the wake can no longer be seen.

The region immediately behind the disc at $y=0$ is shown in more detail in Figure 9. This is the same data as in Figure 8(a), but with different scales on the abscissa and the colorbar. Streamlines have been added in order to better illustrate the backflow region with the twin vortices formed behind the disc in an average perspective. The upper vortex is rotating in the clockwise direction and the lower one in the counterclockwise direction, as indicated by the arrows. A fairly strong backflow is present, where the maximum velocity in the negative streamwise direction is reaching about $35 \%$ of $U_{\text {hub }}$ at around $x / D=0.3$.

\subsection{C2: 2 Discs Positioned 5D Apart in the Streamwise Direction}

The mean streamwise and wall-normal velocity components for the $\mathrm{C} 2$ case are shown in Figure 10 and Figure 11, respectively. Data is shown at the centreline of the wake $(y=0)$ and at a half disc diameter from the centreline in the spanwise direction $(y=0.5 D)$. The discs are positioned at $x=0$ and $x=5 D$. The region around and just behind the discs is a very difficult region to measure accurately, since the flow is highly three-dimensional and the images might be disturbed due to reflections from the discs. The data in this region should therefore be interpreted with great care.

The corresponding turbulence intensities can be seen in Figure 12 (streamwise turbulence intensity, $w_{\mathrm{rms}} / U_{\mathrm{hub}}$ ) and Figure 13 (wall-normal turbulence intensity, $\left.w_{\mathrm{rms}} / U_{\mathrm{hub}}\right)$. Spurious levels of $u_{\mathrm{rms}}$ can be seen just above the discs at $y=0$ (Figure 12(a), which is associated with measurement errors due to reflections. A more homogeneous spread of $u_{\text {rms }}$ can be seen further out in the

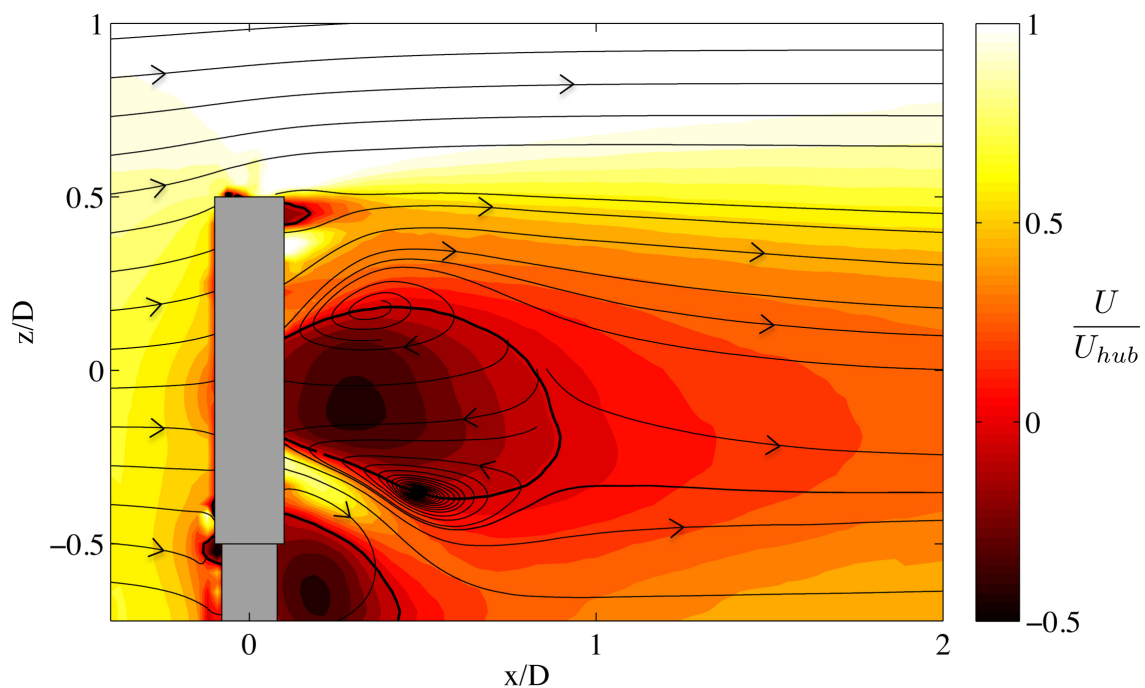

Figure 9. Case C1. Mean streamwise velocity behind the disc at $y=0$. The lines are the 2-dimensional streamlines, with arrows indicating the flow direction. The thicker black lines show the backflow regions, where $U / U_{\text {hub }}<0$. The grey rectangle show the disc location. 


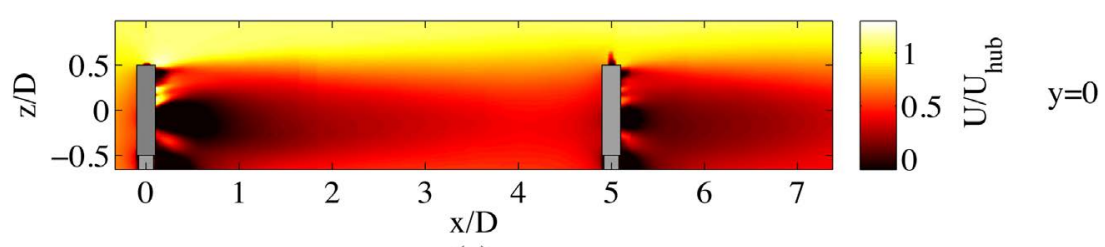

(a)

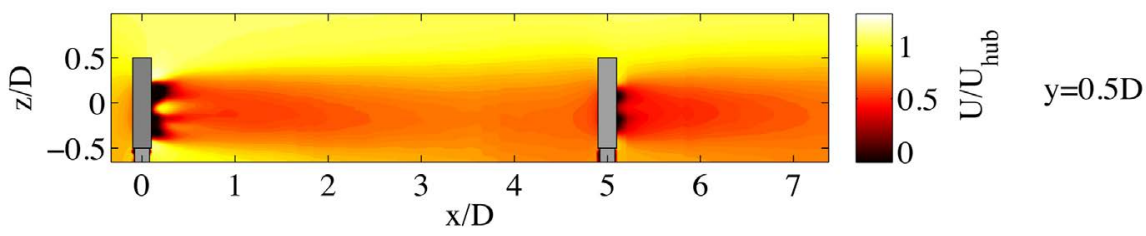

(b)

Figure 10. Case C2. Mean streamwise velocity behind the two discs, positioned at $x / D=0$ and $x / D=5$. The spanwise positions are (a) $y=0$ and (b) $y=0.5 D$. The grey rectangles show the positions of the discs.

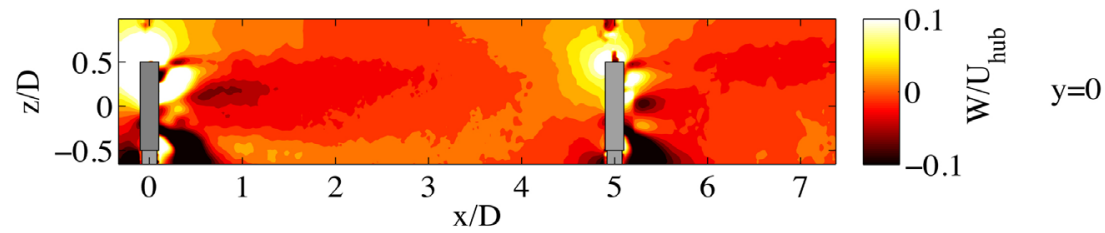

(a)

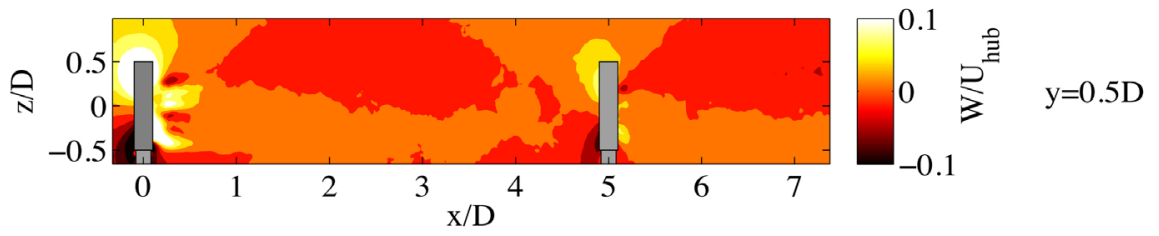

(b)

Figure 11. Case C2. Mean wall-normal velocity behind the two discs, positioned at $x / D=0$ and $x / D=5$. The spanwise positions are (a) $y=0$ and (b) $y=0.5 D$. The grey rectangles show the positions of the discs.

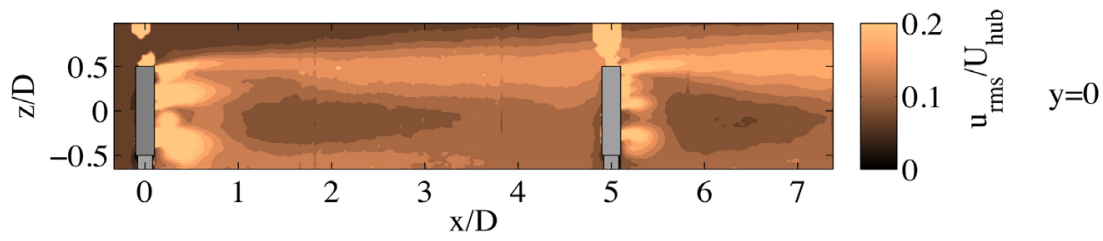

(a)

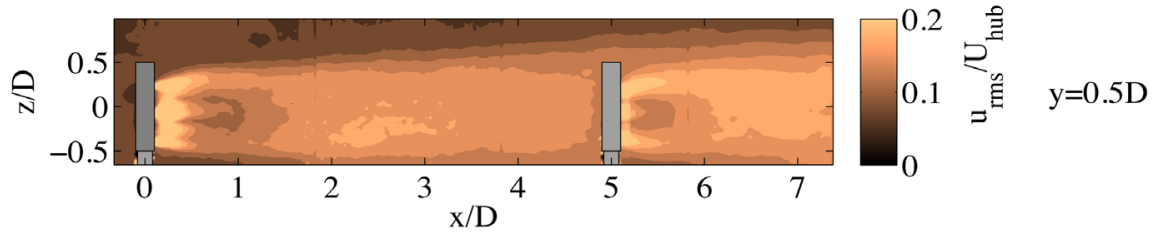

(b)

Figure 12. Case C2. Streamwise turbulence intensity behind the two discs, positioned at $x / D=0$ and $x / D=5$. The spanwise positions are (a) $y=0$ and (b) $y=0.5 D$. The grey rectangles show the positions of the discs. 
wake at $y / D=0.5$ (Figure 12(b)), where instead the disc edge fluctuations are expected to be high in the spanwise direction (note that this component is not measured). The discs generate streamwise and wall-normal turbulence intensities of about $20 \%$ and $10 \%$, respectively. One may observe that the $w_{\text {rms }}$ component is damped relatively quickly in the streamwise direction as compared to the $u_{\mathrm{rms}}$ component.

The inflow conditions for the first and the second disc in C2 are very different, and is shown in Figure 14. This figure displays the mean velocity and turbulence intensity $0.25 D$ upstream of each disc, corresponding to downstream locations of $-0.25 D$ and $4.75 D$, respectively. Note that these profiles are taken with the presence of the discs, and the data has been extracted from Figure 10 and

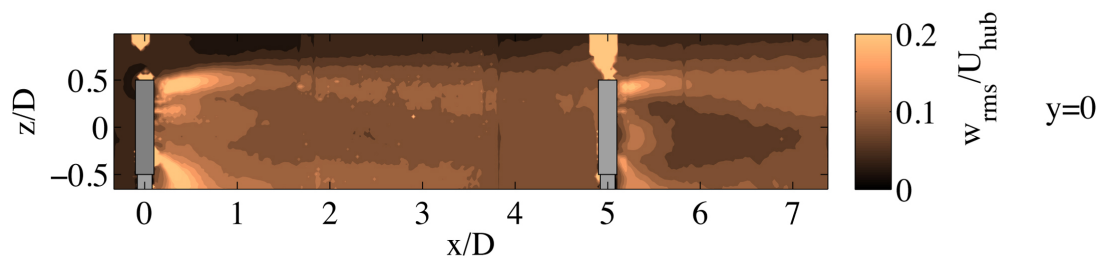

(a)

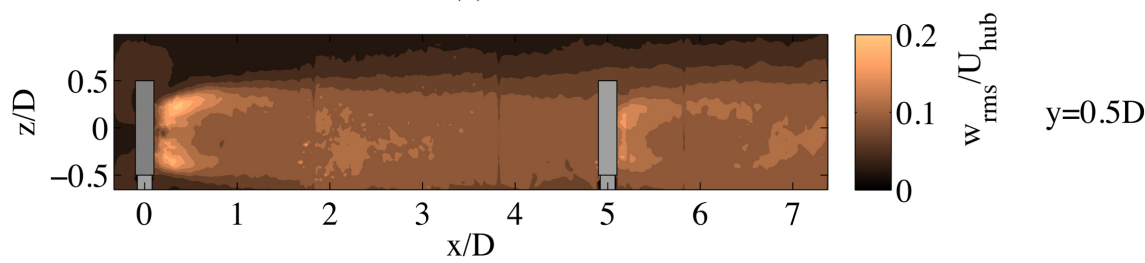

(b)

Figure 13. Case C2. Wall-normal turbulence intensity behind the two discs, positioned at $x / D=0$ and $x / D=5$. The spanwise positions are (a) $y=0$ and (b) $y=0.5 D$. The grey rectangles show the positions of the discs.

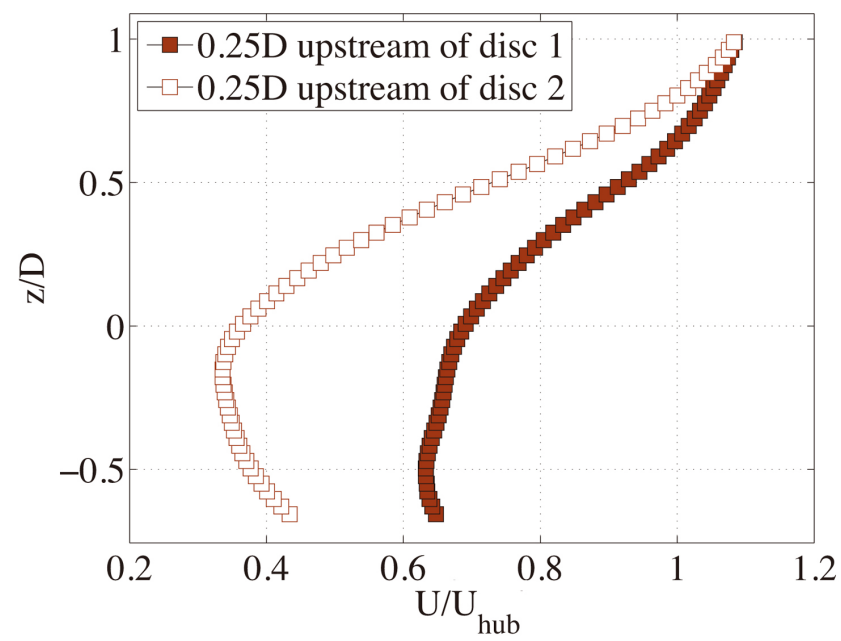

(a)

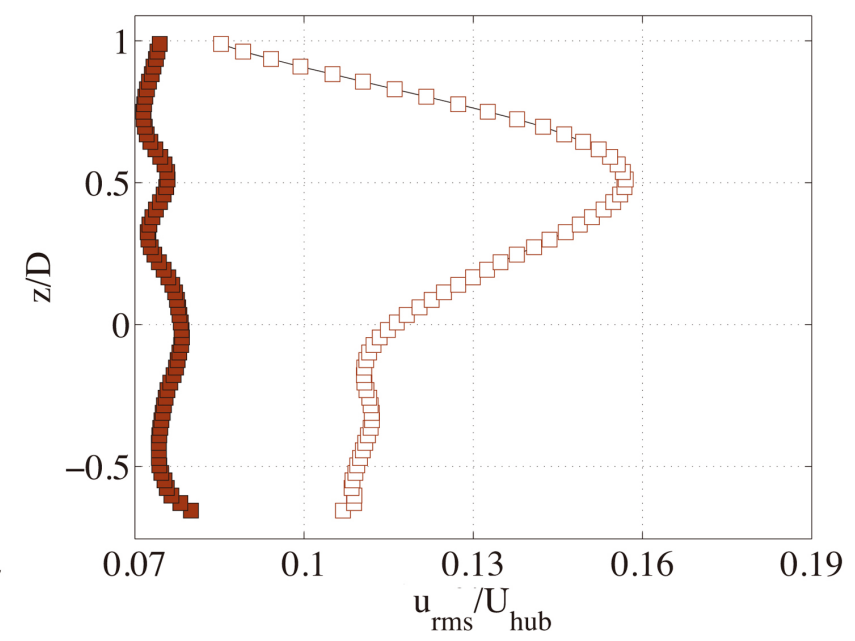

(b)

Figure 14. Case C2. Comparison between the inflow conditions for the two discs (i.e. the flow $0.25 D$ upstream of disc 1 and 2 , which corresponds to $x=-0.25 D$ and $x=4.75 D$, respectively. (a) Mean streamwise velocity and (b) streamwise turbulence intensity. 
Figure 12. Despite the different inlet conditions, with about $50 \%$ of reduced velocity on the centreline upstream of disc 2 and an increase of the maximum streamwise fluctuations by $90 \%$, the mean velocities in the wake behind each disc are fairly similar. This is shown in Figure 15, where the mean streamwise and wall-normal velocity, $2 D$ behind each disc, are plotted. This corresponds to the downstream locations of $2 D$ and $7 D$, respectively. The figure shows three spanwise positions: $y=0$ (centreline), $y=0.5 D$ (middle of the wake, between centreline and edge) and $y=1.1 D$ (edge of the wake). Only fairly small differences can be seen between the two positions, mainly in the streamwise velocity in the inner part of the wake (at $y=0$ and $y=0.5 D$ ), see Figure 15(a) and Figure 15(c). For these two locations, the largest differences occur close to the wall and are in the order of $15 \%$. The differences at the centreline are however negligible. Larger differences can be seen in the corresponding profiles of turbulence intensity, shown in Figure 16. The streamwise turbulence
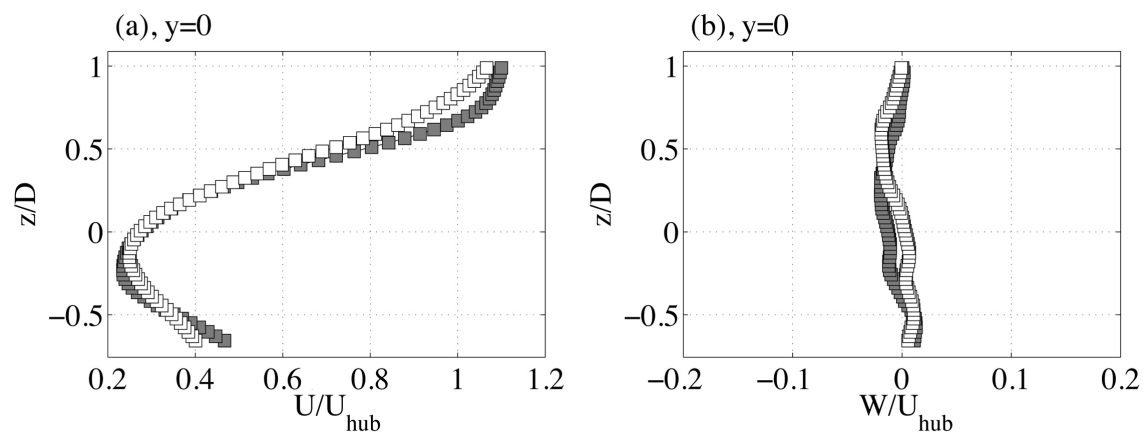

(c), $y=0.5 \mathrm{D}$

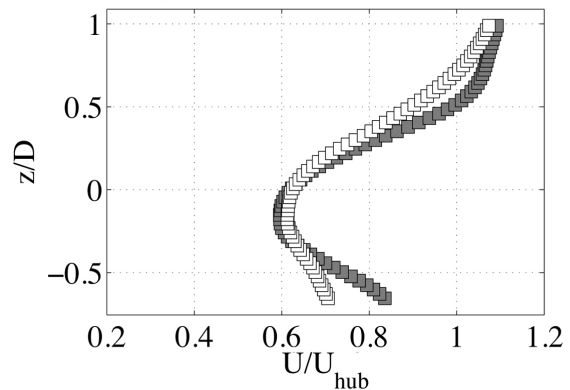

(d), $y=0.5 \mathrm{D}$

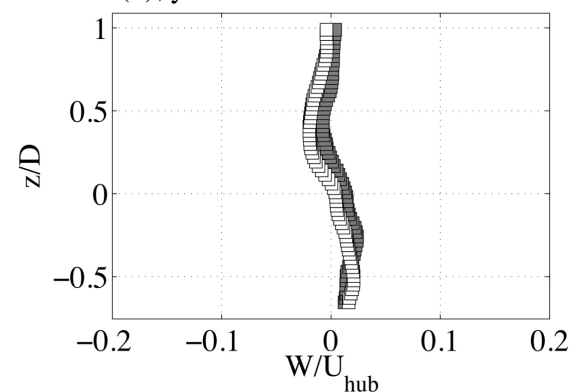

(e), $\mathrm{y}=1.1 \mathrm{D}$
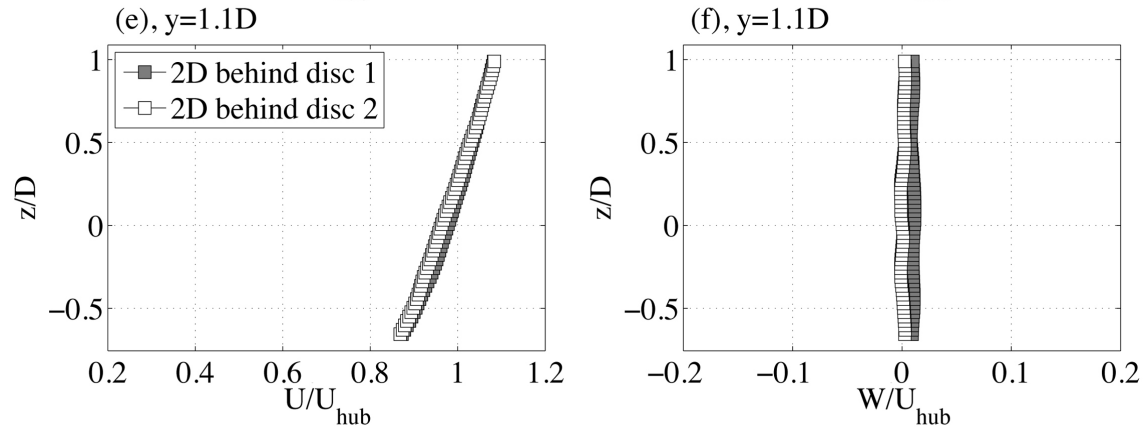

Figure 15. Case C2. Mean streamwise (left column) and vertical (right column) velocity behind disc number 1 and 2. The positions therefore correspond to $x=2 D$ and $x=7 D$. The spanwise locations are from top to bottom: $y=0$ (centreline), $y=0.5 D$ (middle of the wake) and $y=1.1 D$ (edge of the wake). 

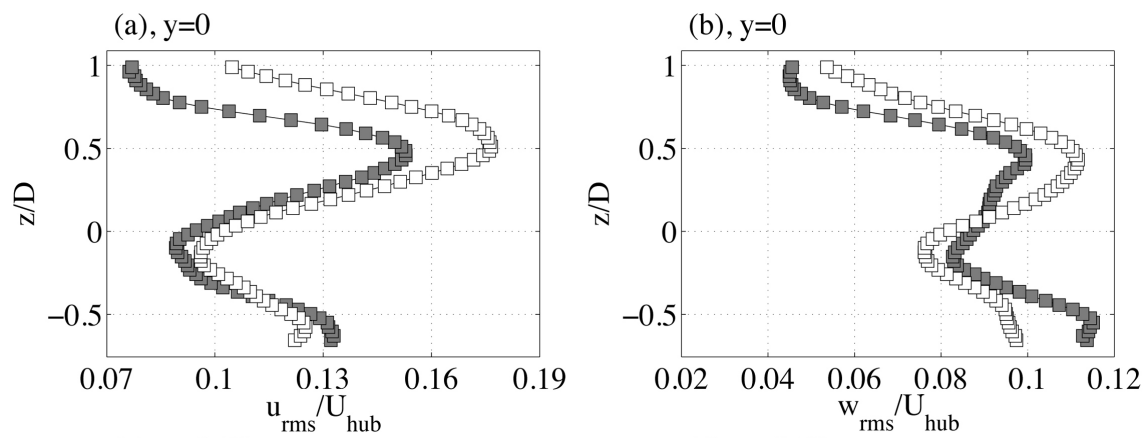

(c), $y=0.5 \mathrm{D}$
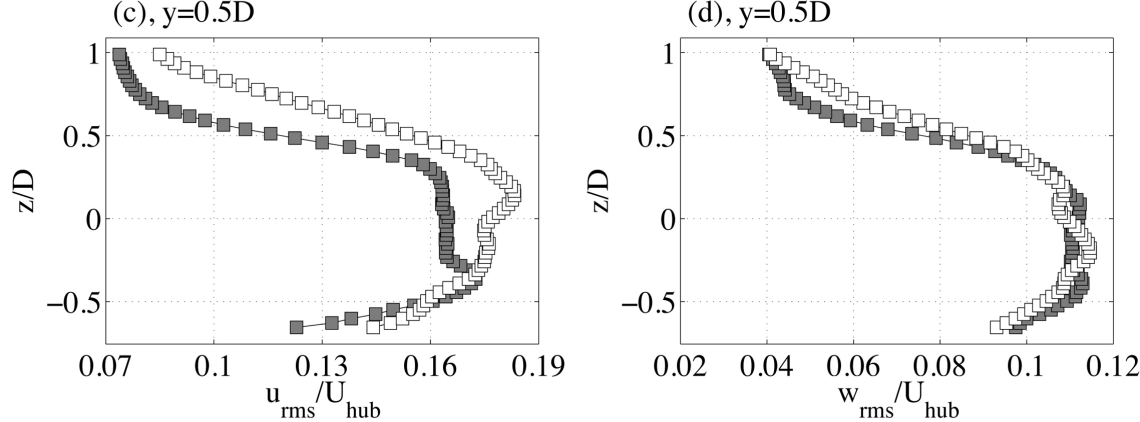

(e), $y=1.1 \mathrm{D}$
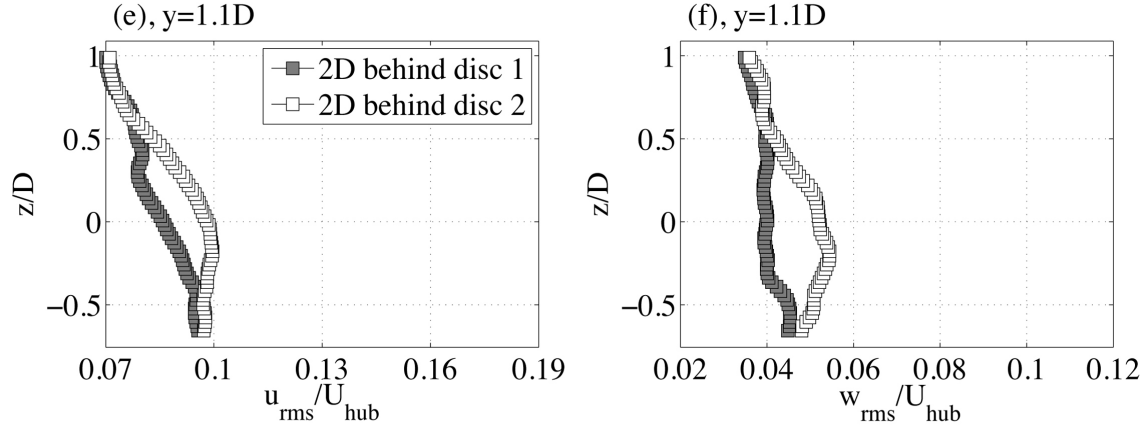

Figure 16. Case C2. Streamwise turbulence intensity (left column) and vertical turbulence intensity (right column) behind disc number 1 and 2. The positions therefore correspond to $x=2 D$ and $x=7 D$. The spanwise locations are from top to bottom: $y=0$ (centreline), $y=0.5 D \quad$ (middle of the wake) and $y=1.1 D \quad$ (edge of the wake).

intensity is generally higher behind the second disc (Figure 16(a), Figure 16(c) and Figure 16(e)). At the edges of the discs, at $z / D=0.5$, the difference is around $20 \%$ in the inner part of the wake. For the wall-normal turbulence intensity, a somewhat different behaviour can be seen. At the centreline ( $y=0$, Figure 16(b)), the turbulence behind disc 2 has a wider spread straight behind the disc (in the region $-0.5<z / D<0.5$ ). In the middle of the wake ( $y=0.5 D$, Figure 16(d)), no significant difference can be seen between the cases, but at the edge of the wake $(y=1.1 D$, Figure 16(f)), the turbulence is higher behind $\operatorname{disc} 2$.

\subsection{Varying the Distance to the Second Disc}

A detailed comparison between all three cases ( $1,1, \mathrm{C} 2$ and $\mathrm{C} 3)$ is shown in Figure 17. The figure shows wall-normal profiles at $x=2.5 \mathrm{D}$ and $x=3.5 \mathrm{D}$. Four spanwise positions are shown from top to bottom: $y=0, y=0.5 D$, 

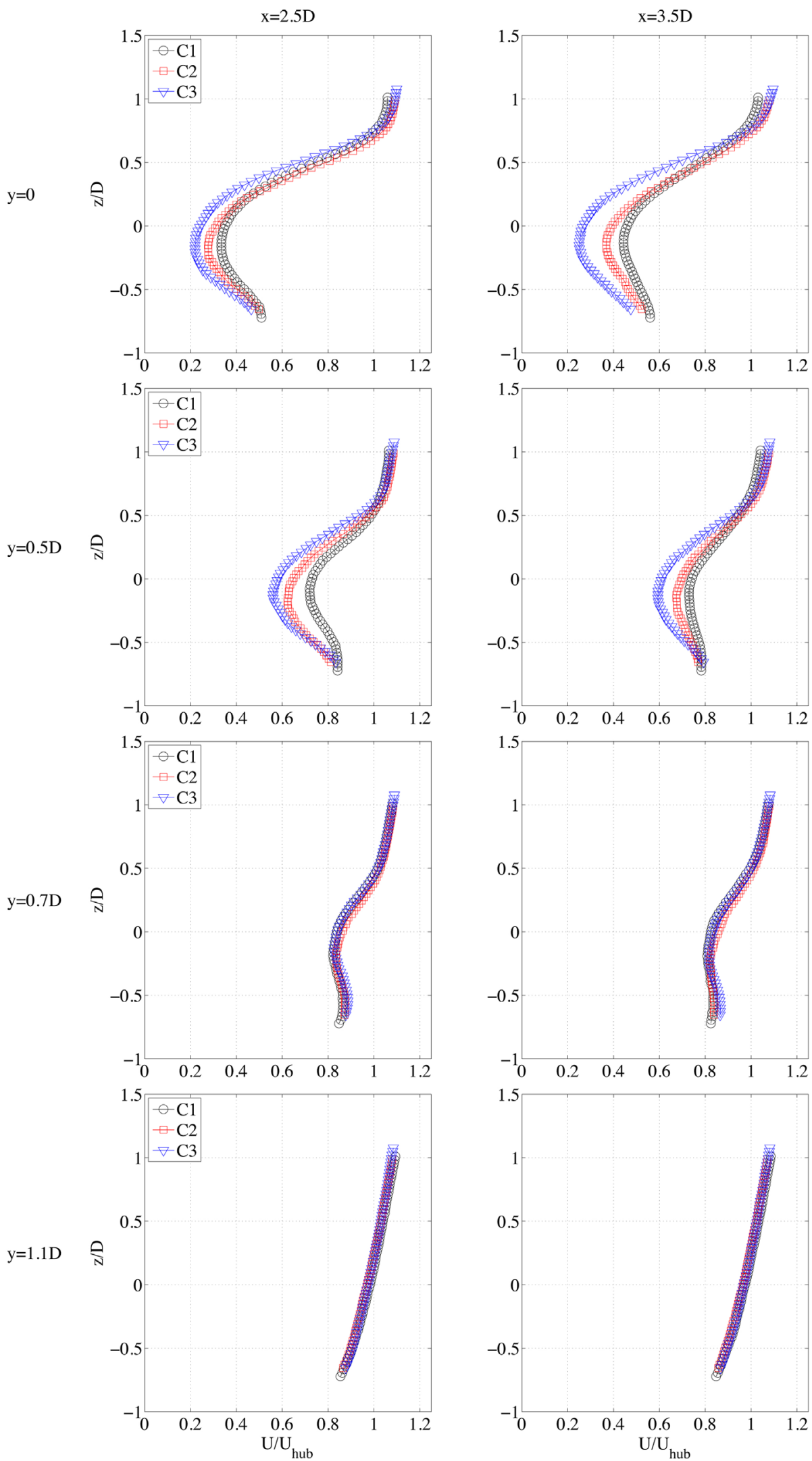

Figure 17. Case C1, C2 and C3. Mean streamwise velocity in the wake. From top to bottom: $y=0, y=0.5 D, y=0.7 D$ and $y=1.1 D$. 
$y=0.7 D$ and $y=1.1 D$. For the inner part of the wake $(y=0$ and $y=0.5 D)$, it can be seen that the velocity is lower for C3, especially closer to the second disc, at $x=3.5 D$. On the centre line $(y=0)$ at $x / D=3.5$ the minimum velocity in the wake for the $\mathrm{C} 2$ and $\mathrm{C} 3$ cases are reduced by 21 and $46 \%$, respectively, with respect to the $\mathrm{C} 1$ case. This reduction is expected, since it is likely that the upstream influence of the second disc will be stronger approaching the second disc. These differences can however not be seen further out in the wake in the spanwise direction $(y=0.7 D$ and $y=1.1 D)$. The streamwise turbulence intensity in Figure 18 shows expected maxima around $x / D=0.5$, i.e. in the region of strongest mean velocity shear. As the mean wake profile changes, by moving out from the wake in the spanwise direction, the turbulence intensity profiles approaches the classical distribution of a far field wake with a single peak at the largest velocity deficit. Apparently, it is the mere presence of the second disc that affects the turbulence in this region. For the outer part $(y=0.7 D$ and $y=1.1 D)$, all three curves are close to each other, showing that there is no significant difference between the three cases in this region.

Figure 19(a) shows the downstream development of the streamwise velocity at the centreline, $y=z=0$. The fastest wake recovery is seen for C1 with only one disc. Due to the closer distance between the discs for the C3 case, the velocity recovery rate is lower compared to case $\mathrm{C} 2$ in the region $2 \leq x / D \leq 3.5$. Due to this and the longer recovery for the $\mathrm{C} 2$ case, the velocity recovery is 55\% larger than for the $\mathrm{C} 3$ case with respect to the stagnation point. In order to be able to compare the two cases, Figure 19(b) shows the same data but with C3 shifted $1 D$ downstream. One may observe that the difference in velocity upstream of the second disc is kept downstream. The difference between $\mathrm{C} 2$ and C3 $1 D$ upstream of disc 2 (at $x / D=4$ in this figure) is around $45 \%$, which is the same difference as $1 D$ downstream of disc 2 (at $x / D=6$ in this figure). After the second disc, the wake recovery has the same slope, i.e. the same recovery rate. A fairly large backflow can be seen behind the discs, ranging from approximately $15 \%$ to $35 \%$ of $U_{\text {hub }}$. These local minima occur about $0.2 D-0.3 D$ behind the discs. Comparing the backflow regions of the second disc in $\mathrm{C} 2$ and $\mathrm{C} 3$ with the backflow region in the $\mathrm{C} 1$ case, one may conclude that both the maximum backflow and streamwise extent increases with hub height velocity.

\section{Summary and Conclusions}

Particle image velocimetry and static pressure measurements of the wake flow behind two perforated discs have been performed in a wind tunnel. The discs have a diameter $D=45 \mathrm{~mm}$ and a thrust coefficient $C_{T}=0.85$. The discs were positioned in three different configurations: a reference case with one disc (case C1), with the disc $5 D$ apart in the streamwise direction (case C2) and with the discs $4 D$ apart in the streamwise direction (case $\mathrm{C} 3$ ).

For $\mathrm{C} 2$, the inlet conditions for the two discs (i.e. the flow $0.25 \mathrm{D}$ upstream of 

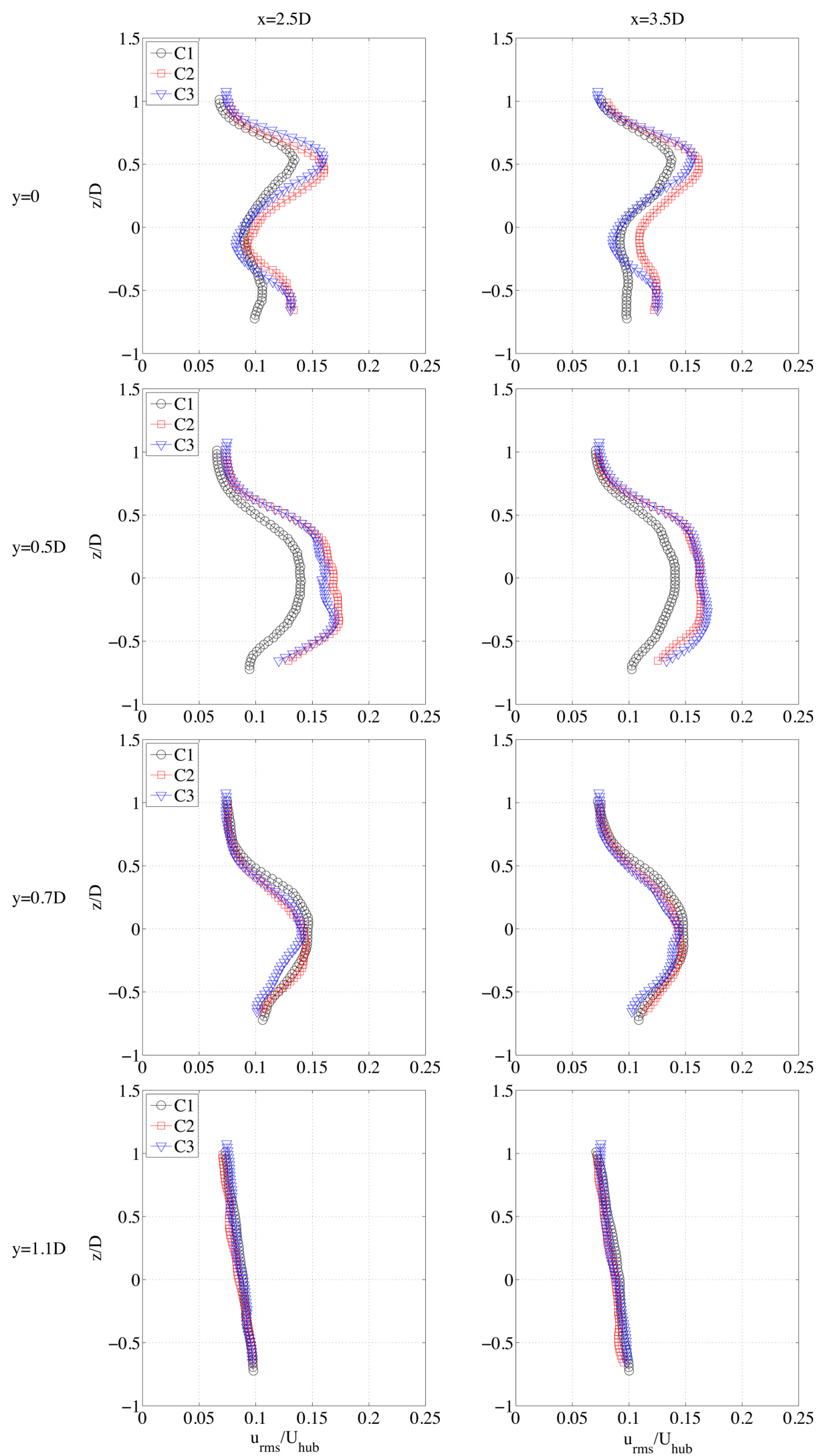

Figure 18. Case C1, C2 and C3. Streamwise turbulence intensity in the wake. From top to bottom: $y=0, y=0.5 D, y=0.7 D$ and $y=1.1 D$. 


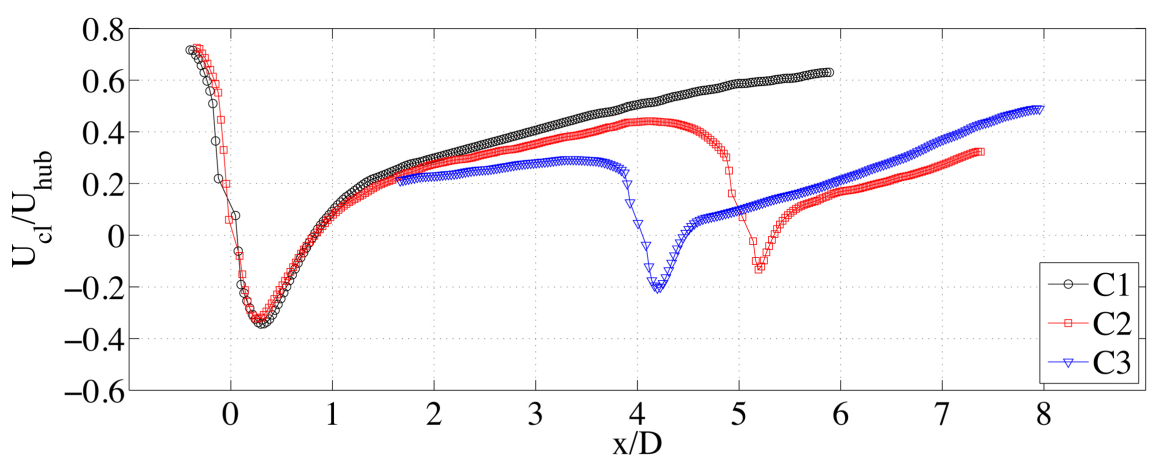

(a)

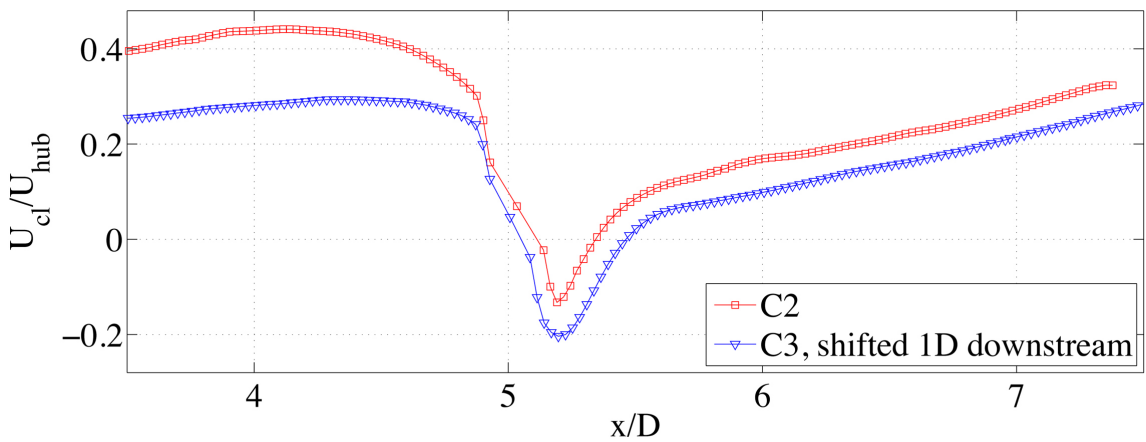

(b)

Figure 19. Downstream development of the minimum velocity in the wake. In (b), C3 has been shifted $1 D$ downstream in order to compare with $\mathrm{C} 2$.

each disc) were significantly different: the mean velocity at the centreline was reduced with about $50 \%$ and the maximum streamwise fluctuation was increased by $90 \%$ for disc 2 , as compared to disc 1 . Despite the different inlet conditions, the mean velocity $2 D$ behind each disc remained fairly similar when scaled with the hub height velocity. The turbulence intensities were however in general higher behind the second disc, the difference was around $20 \%$ at the upper edge of the disc. Decreasing the distance between the discs from $5 D$ to $4 D$ seemed to have a somewhat larger effect on the mean velocity than on the turbulence intensity. The presence of the second disc was also found to have a significant effect on the wake recovery, already at $1.5 \mathrm{D}$ downstream of the most upstream disc.

The pressure distribution at ground level in the wake of a wind turbine, showing the pressure footprint ( $\mathrm{p}-\mathrm{f}$ ) of the model turbine, is believed to provide valuable information that can be used for both qualitative and quantitative wake analyses. Detailed information of the pressure and the velocity fields together can be utilized to understand the intricacies of the complex phenomena of wake interaction with good fidelity. In this investigation we show that, after an initial drop in size of the wake footprints when a second disc is added closely behind a first disc, the wake region of the downstream disc is a factor of two greater than the upstream one provided that the mutual distance between the turbine models is in the range $\Delta x / D \lesssim 5$. We can also conclude that, after about $\Delta x / D=5$, the wake footprints increase as the mutual distance is increased and for very large 
mutual distances, approximately $\Delta x / D>15$, the footprint of the downstream wind turbine has recovered and is about the same as for single wind turbine.

We believe that the $\mathrm{p}-\mathrm{f}$ method provides valuable pictures of the static pressure field on ground. The resolution depends on the number of taps and inter-tap spacing i.e. number of taps per unit surface area of the plate. This method is simple, non-intrusive, economical and a fast measuring technique that can be used for field measurements using microbarometers. This technique provides pictures of the wake in the $x y$-plane (i.e. horizontal plane) and thus regions with strong interaction can be identified immediately.

Future experiments with a higher spatial resolution of the static pressure measurements as well as the PIV measurements are planned, with the main aim to find a correlation relation between the ground pressure and the velocity deficit in the wake behind wind turbine models. In order to develop such a correlation relation it would be favorable to measure in a uniform stream across the wind turbine model and not in shear layer as have been done in the present investigation.

\section{Acknowledgements}

University of Gävle is acknowledged for funding the project. We thank the workshop technicians and research engineer Leif Claesson at the Atmospheric Boundary Layer wind tunnel, University of Gävle, for their valuable help during the experiments.

\section{References}

[1] Vermeer, L.J., Sørensen, J.N. and Crespo, A. (2003) Wind Turbine Wake Aerodynamics. Progress in Aerospace Sciences, 39, 467-510. https://doi.org/10.1016/S0376-0421(03)00078-2

[2] Sørensen, J.N. (2011) Aerodynamic Aspects of Wind Energy Conversion. Annual Review of Fluid Mechanics, 43, 427-428. https://doi.org/10.1146/annurev-fluid-122109-160801

[3] Hansen, M.O.L., Sørensen, J.N., Voutsinas, S., Sørensen, N. and Madsen, H.A. (2006) State of the Art in Wind Turbine Aerodynamics and Aeroelasticity. Progress in Aerospace Sciences, 42, 285-330. https://doi.org/10.1016/j.paerosci.2006.10.002

[4] Yang, X., Kang, S. and Sotiropoulos, F. (2012) Computational Study and Modeling of Turbine Spacing Effects in Infinite Aligned Wind Farms. Physics of Fluids, 24, 107-115.

[5] Rind, E. and Castro, I.P. (2012) On the Effects of Free-Stream Turbulence on Axisymmetric Disc Wakes. Experiments in Fluids, 53, 301-318. https://doi.org/10.1007/s00348-012-1288-2

[6] Wu, Y. and Porté-Agel, F. (2013) Simulation of Turbulent Flow inside and above Wind Farms: Model Validation and Layout Effects. Boundary-Layer Meteorology, 146, 181-205. https://doi.org/10.1007/s10546-012-9757-y

[7] Storey, R.C., Norris, S.E. and Cater, J.E. (2014) An Actuator Sector Method for Efficient Transient Wind Turbine Simulation. Wind Energy, 18, 699-711. https://doi.org/10.1002/we.1722 
[8] Cal, R.B., Lebrón, J., Castillo, L., Kang, H.S. and Meneveau, C. (2010) Experimental Study of the Horizontally Averaged Flow Structure in a Model Wind-Turbine Array Boundary Layer. Journal of Renewable and Sustainable Energy, 2, 103-106.

[9] España, G., Aubrun, S., Loyer, S. and Devinant, P. (2011) Spatial Study of the Wake Meandering using Modelled Wind Turbines in a Wind Tunnel. Wind Energy, 14, 923-937. https://doi.org/10.1002/we.515

[10] España, G., Aubrun, S., Loyer, S. and Devinant, P. (2012) Wind Tunnel Study of the Wake Meandering Downstream of a Modelled Wind Turbine as an Effect of Large Scale Turbulent Eddies. Journal of Wind Engineering and Industrial Aerodynamics, 101, 24-33. https://doi.org/10.1016/j.jweia.2011.10.011

[11] Aubrun, S., Loyer, S., Hancock, P.E. and Hayden, P. (2013) Wind Turbine Wake Properties: Comparison between a Non-Rotating Simplified Wind Turbine Model and a Rotating Model. Journal of Wind Engineering \& Industrial Aerodynamics, 120, 1-8. https://doi.org/10.1016/j.jweia.2013.06.007

[12] Castro, I.P. (1971) Wake Characteristics of Two-Dimensional Perforated Plates Normal to an Air-Stream. Journal of Fluid Mechanics, 46, 599-609. https://doi.org/10.1017/S0022112071000727

[13] Cannon, S., Champagne, F. and Glezer, A. (1993) Observations of Large-Scale Structures in Wakes Behind Axisymmetric Bodies. Experiments in Fluids, 14, 447-450. https://doi.org/10.1007/BF00190199

[14] Miau, J.J., Leu, T.S., Liu, T.W. and Chou, J.H. (1997) On Vortex Shedding behind a Circular Disk. Experiments in Fluids, 23, 225-233.

https://doi.org/10.1007/s003480050106

[15] Medici, D. (2005) Experimental Studies of Wind Turbine Wakes-Power Optimisation and Meandering. PhD Thesis, KTH Royal Institute of Technology, Stockholm.

[16] Sforza, P.M., Sheerin, P. and Smorto, M. (1981) Three Dimensional Wakes of Simulated Wind Turbines. AIAA Journal, 19, 1101-1107. https://doi.org/10.2514/3.60049

[17] Khan, M.A., Sandberg, M. and Fransson, J.H.M. (2013) On the Wake Pressure Footprint for Wake Flow Analysis. Proceedings of the Annual Conference of European Wind Energy Association.

[18] Chen, W.F. and Lui, E.M. (2005) Handbook of Structural Engineering. CRC Press. https://doi.org/10.1201/9781420039931 